\title{
Unknown inputs observers for state and unknown inputs estimation in a class of discrete-time Lipschitz nonlinear systems
}

\author{
Ben Warrad Seifeddine, Dhahri Slim and Ben Hmida Fayçal \\ Research unit C3S, ESSTT, University of Tunis, 5 av. Taha Hussein BP $56-1008$ Tunis, Tunisia \\ E-mails: ben.warrad_seifeddine@hotmail.com,slim_dhahri@yahoo.fr, faycal.benhmida@esstt.rnu.tn
}

\begin{abstract}
In this paper, we propose a method for estimating of state and unknown inputs based in observers approach in a class of discrete-time Lipschitz nonlinear systems with unknown inputs both state and measurement. This method design is obtained using a particular Lyapunov function in order to guaranty the asymptotic stability of the error estimation. The observer gains are determined in terms of Linear Matrix inequalities (LMI). Performances of the proposed approach are illustrated through simulation results.
\end{abstract}

Keywords: nonlinear systems, Lipschitz systems, discrete-time, extended unknown inputs observers, unknown disturbances, state estimation, unknown inputs estimation.

\section{INTRODUCTION}

During the past decades, Luenberger observer has been extended to continuous-time linear systems with unknown inputs. The problem is of great importance in practice since there are many situations where the disturbances or inputs are inaccessible.

Many complete results on these observers can be found in the literature ([1], [2], [3], [4], [5], [ 6] and [7]) and it is rare the papers ([8] and [9]) that deal with the problem of designing of unknown inputs observers for a discrete-time linear system. However, the problem of the state and unknown inputs estimation of a nonlinear systems has been widely studied in the past few years. In fact, knowledge of the state of system is needed in many applications. In the context enormous research activities have been developed to deal with the design of extended unknown inputs observers for nonlinear systems. This initiates the need to word the state an unknown inputs estimation as they are regarded as an indispensable unit for several applications; such as, FDI: Fault Detection and Isolation ([10] and [11]).

As we know, however, it is often difficult or even impossible to design a state observer and unknown inputs observers for a general nonlinear system. Therefore, up to now, most of the works on observer synthesis were carried out for some special classes of nonlinear systems; for instance, observes for Lipschitz nonlinear systems.

Thau [12] first studied an observer design of Lipschitz nonlinear systems and proposed a sufficient condition ensuring the asymptotic stability of the observer error dynamics. Even though, this condition can be useful for the observer observation analysis, it cannot ensure its stability.
Consequently, other works have been interested in the design of extended unknown inputs observers for Lipschitz nonlinear systems.

Unfortunately, most of the works presented in the literature deal with continuous-time Lipschitz systems ([13], [14], [15], [16] and [17]). Indeed, there are few papers ([18] and [19]) dealing with discrete-time Lipschitz systems.

The observer synthesis condition presented by Zemouche et al. ([18] and [19]) is obtained by injecting the Lipschitz condition into the classical Lyapunov stability inequality. This condition restricts the applicability of this approach to the particular class of Lipschitz nonlinear systems. Indeed, there are few papers ([20] and [21]) dealing with discrete time observers. The authors of the above works used estimation error that boils down to solving a large number of Lyapunov equations.

This paper is organized as follows; In section 2, the problem statement and some preliminaries are mentioned. In section 3 , we propose a procedure design achieving the convergence of the estimation error towards zero and for each procedure we use a particular new Lyapunov function. In section 4, we propose to study the extended UIO design for state and unknown input estimation. In section 5, a simulation example of a single-link robotic arm with a revolute elastic joint is described illustrating the effectiveness of th our proposed procedure. Finally, section 6 presents some concluding remarks.

\section{PRELIMINARIES AND PROBLEM STATEMENT}

Consider the class of discrete-time nonlinear system described by

$$
\left\{\begin{array}{l}
x_{k+1}=A x_{k}+B u_{k}+g\left(x_{k}\right) \\
y_{k}=C x_{k}
\end{array}\right.
$$

where $k \in \aleph, x_{k} \in \Re^{n}$ is the state vector, $u_{k} \in \Re^{l}$ is the control input vector and $y_{k} \in \Re^{m}$ is the output vector $A, B$ and $C$ are constant matrices of appropriate dimensions. The term $g: \Re^{n} \rightarrow \Re^{l}$ is a nonlinear function. We assume that $(A, C)$ is observable and $C$ has full row rank.

For system (1), the following assumptions are made available: 


\section{Assumption 1:}

There exists a constant $\gamma>0$ such that for all $x_{k}, \hat{x}_{k} \in \Re^{n}$.

$$
\left\|g\left(x_{k}\right)-g\left(\hat{x}_{k}\right)\right\|_{2} \leq \gamma\left\|x_{k}-\hat{x}_{k}\right\|_{2}
$$

where $\gamma$ is the Lipschitz constant.

Given the system (1) we investigate in this paper the synthesis of an observer with the following structure

$$
\hat{x}_{k+1}=A \hat{x}_{k}+B u_{k}+g\left(\hat{x}_{k}\right)+K\left(y_{k}-C \hat{x}_{k}\right)
$$

where $\hat{x}_{k}$ denotes the estimate of the state vector $x_{k}$. Our aim is to find the gain $K$ knowing that the estimation error

$$
e_{k}=x_{k}-\hat{x}_{k}
$$

converges asymptotically towards zero.

using (1) and (3), the dynamic of the estimation error is given by

$$
e_{k+1}=A_{k} e_{k}+r_{k}
$$

where $r_{k}=g\left(x_{k}\right)-g\left(\hat{x}_{k}\right)$ and $A_{k}=A-K C$.

In the following, thanks to particular Lyapunov functions, we introduce a set of results that consist of sufficient conditions for synthesis of the observer gain $K$.

\section{SYNTHESIS OF THE OBSERVER}

Here, we introduce our main work which consists in new LMI feasibility conditions for the observer problem design. In follows, we present a theorem which proposes a sufficient condition achieving the convergence of the estimation error towards zero. The stability analysis is performed by the use of a particular new Lyapunov function.

\section{Theorem 1:}

The state estimation error (5) converges asymptotically to the origin of the Lipschitz constant $\gamma$ satisfies $\gamma<$ $\frac{\underline{\underline{\sigma}\left(Q^{(1 / 2)}\right)}}{\sqrt{\bar{\sigma}\left(Q^{(-1 / 2)} A_{k}^{T} P\right)+\bar{\sigma}(P)+\bar{\sigma}\left(Q^{(-1 / 2)} A_{k}^{T} P\right)}}$. where $P=P^{T}>0$
$, L, \alpha>0$ and $Q-0.5 P>0$ solution of the following

$$
\begin{gathered}
{\left[\begin{array}{cc}
0.5 P-\gamma^{2} \alpha I & A^{T} P-C^{T} L^{T} \\
P A-L C & P
\end{array}\right]>0} \\
{\left[\begin{array}{cc}
\alpha I & P \\
P & \alpha I
\end{array}\right]>0}
\end{gathered}
$$

The observer gain $K$ is given by $K=P^{-1} L$.

Proof: Consider the Lyapunov function

$$
V_{k+1}=e_{k+1}^{T} P e_{k+1} \quad, \quad P \in \Re^{n \times n}
$$

where $P=P^{T}>0$.

According to the Lyapunov theorem, the observer (3) converges asymptotically if

$$
\Delta V=V_{k+1}-V_{k}<0
$$

By subsisting (5) and (8) in (9), we have

$$
\Delta V=e_{k}^{T}\left(A_{k}^{T} P A_{k}-P\right) e_{k}+2 e_{k}^{T} A_{k}^{T} P r_{k}+r_{k}^{T} P r_{k}<0
$$

Let $P=P^{T}>0$, be a solution of the following Lyapunov equation

$$
Q=P-A_{k}^{T} P A_{k}
$$

with $A_{k}$ being a stable matrix and $Q^{T}=Q>0$.

\section{Lemma 1 [22]}

For any matrices $X, Y \in \Re^{n}$ with appropriate dimensions and any positive-definite matrix $P \in \Re^{n \times n}$, we have

$$
2 X^{T} Y<X^{T} X+Y^{T} Y
$$

Applying the lemma 1, we obtain

$$
2 e_{k}^{T} A_{k}^{T} P r_{k} \leq e_{k}^{T} A_{k}^{T} P A_{k} e_{k}+r_{k}^{T} \operatorname{Pr}_{k}
$$

Substituting (13) into (10) gives

$$
\Delta V \leq 2 e_{k}^{T}\left[A_{k}^{T} P A_{k}-0.5 P\right] e_{k}+2 r_{k}^{T} P r_{k}<0
$$

On the other hand, from the Lipschitz condition (2) we deduced that

$$
r_{k}^{T} P r_{k} \leq \gamma^{2} \bar{\sigma}(P) e_{k}^{T} e_{k}
$$

Inserting (15) into (14) yields

$$
\Delta V \leq 2 e_{k}^{T}\left[\gamma^{2} \bar{\sigma}(P) I-\left(0.5 P-A_{k}^{T} P A_{k}\right)\right] e_{k}<0
$$

Substituting (11) into (16) gives

$$
\Delta V \leq 2 e_{k}^{T}\left[\gamma^{2} \bar{\sigma}(P) I-(Q-0.5 P)\right] e_{k}<0
$$

which is equivalent to

$$
\gamma<\sqrt{\frac{1}{\bar{\sigma}(P)} \frac{e_{k}^{T}(Q-0.5 P) e_{k}}{e_{k}^{T} e_{k}}}
$$

Using Rayleigh and Schwartz inequalities [23], we have

$$
\frac{e_{k}^{T}(Q-0.5 P) e_{k}}{e_{k}^{T} e_{k}} \geq \underline{\sigma}(Q-0.5 P)
$$

Therefore, inequality (18) can be written in the following form

$$
\gamma<\sqrt{\frac{\sigma(Q-0.5 P)}{\bar{\sigma}(P)}} \text { with } Q-0.5 P>0
$$

Now, it can be shown that (16) is equivalent to

$$
0.5 P-\gamma^{2} \bar{\sigma}(P) I-A_{k}^{T} P A_{k}>0
$$

Suppose that $\bar{\sigma}(P)<\alpha$ with $\alpha>0 . \bar{\sigma}(P)<\alpha$ is equivalent to $\alpha-\alpha^{-1} P P>0$ which can be written in the following LMI

$$
\left[\begin{array}{cc}
\alpha I & P \\
P & \alpha I
\end{array}\right]>0 \quad \alpha>0 \quad P>0
$$

By applying, the Schur complement in (21), we obtain:

$$
\left[\begin{array}{cc}
0.5 P-\gamma^{2} \alpha I & A_{k}^{T} \\
A_{k} & P^{-1}
\end{array}\right]>0
$$

which is equivalent to

$$
\left[\begin{array}{cc}
0.5 P-\gamma^{2} \alpha I & A_{k}^{T} P \\
P A_{k} & P
\end{array}\right]>0
$$


Using the change of the variable $K=P^{-1} L$ into (24) gives the result LMI (6). It is now straightforward to see all of the design procedure summarized as follows:

Step 1: Solve a set of LMIs: (6) and (7).

Step 2: Obtain the gain matrix $K=P^{-1} L$.

Step 3: Test the converge condition (20).

\section{EXTENDED UIO DESIGN FOR STATE AND UNKNOWN INPUT ESTIMATION}

\section{A. Extended UIO design for state estimation}

Consider the discrete-time Lipschitz system

$$
\left\{\begin{array}{l}
x_{k+1}=A x_{k}+B u_{k}+g\left(x_{k}\right)+E d_{k} \\
y_{k}=C x_{k}+F d_{k}
\end{array}\right.
$$

where $x_{k} \in \Re^{n}$ is the state vector, $y_{k} \in \Re^{m}$ is the output vector, $u_{k} \in \Re^{l}$ is the known input vector and $d_{k} \in \Re^{q}$ is the unknown input (or disturbance) vector. $A, B, C, E$ and $F$ are known matrices with appropriate dimensions.

The aim of extended unknown inputs observers is to produce an estimation despite the presence of unknown inputs.

It is possible to simplify the measurement equation using a transformation below:

we assume $T_{y}=\left(I-F F^{+}\right)$with $F^{+}=\left(F^{T} F\right)^{-1} F^{T}$ such as $T_{y} F=\left(I-F F^{+}\right) F=0$.

Indeed, the system (25) discrete-time Lipschitz nonlinear systems became as follows

$$
\left\{\begin{array}{l}
x_{k+1}=A x_{k}+B u_{k}+g\left(x_{k}\right)+E d_{k} \\
\bar{y}_{k}=C_{y} x_{k}
\end{array}\right.
$$

For system (26), the following assumptions are made available: Assumption 2:

$$
\operatorname{rank}\left(C_{y} E\right)=\operatorname{rank}(E)
$$

\section{Assumption 3:}

The invariant zeros of the triplet $\left(A, E, C_{y}\right)$ are stable that is $\operatorname{rank}\left[\begin{array}{cc}\lambda I-A & E \\ C_{y} & 0\end{array}\right]=n+\operatorname{rank}(E) \quad \lambda \in \mathbb{C}$ with $|\lambda| \geq 1$ If the assumption 3 is satisfied, then it is possible to define a generalized inverse of a matrix $\left(C_{y} E\right)$ as a matrix $\left(C_{y} E\right)^{+} \in$ $\Re^{n \times m}$ such that $\left(C_{y} E\right)\left(C_{y} E\right)^{+}\left(C_{y} E\right)=\left(C_{y} E\right)$

Now, to design extended unknown inputs observer, we use the measurement equation:

$$
\bar{y}_{k+1}=C_{y} x_{k+1} \Leftrightarrow\left(C_{y} E\right)^{+} \bar{y}_{k+1}=\left(C_{y} E\right)^{+} C_{y} x_{k+1}
$$

which is equivalent to

$$
\left(C_{y} E\right)^{+} \bar{y}_{k+1}=\left(C_{y} E\right)^{+} C_{y}\left[A x_{k}+B u_{k}+g\left(x_{k}\right)+E d_{k}\right]
$$

where we can rewrite (28) as follows

$$
d_{k}=\left(C_{y} E\right)^{+} \bar{y}_{k+1}-\left(C_{y} E\right)^{+} C_{y}\left[A x_{k}+B u_{k}+g\left(x_{k}\right)\right]
$$

Substituting (29) into (26) gives

$$
x_{k+1}=A_{1} x_{k}+B_{1} u_{k}+g_{1}\left(x_{k}\right)+H \bar{y}_{k+1}
$$

with

$$
\begin{gathered}
A_{1}=\left[I-E\left(C_{y} E\right)^{+} C_{y}\right] A B_{1}=\left[I-E\left(C_{y} E\right)^{+} C_{y}\right] B \\
H=E\left(C_{y} E\right)^{+} g_{1}\left(x_{k}\right)=\left[I-E\left(C_{y} E\right)^{+} C_{y}\right] g\left(x_{k}\right)
\end{gathered}
$$

Thus, the extended unknown input observer for (26) is given as follows

$$
\hat{x}_{k+1}=A_{1} \hat{x}_{k}+B_{1} u_{k}+g_{1}\left(\hat{x}_{k}\right)+H \bar{y}_{k+1}+K\left(\bar{y}_{k}-C_{y} \hat{x}_{k}\right)
$$

Therefore, the extended unknown input observer can be written in the following form

$$
\begin{aligned}
\hat{x}_{k+1}= & {\left[I-E\left(C_{y} E\right)^{+} C_{y}\right] \hat{x}_{k+1 / k}+E\left(C_{y} E\right)^{+} \bar{y}_{k+1} } \\
& +K\left(\bar{y}_{k}-C_{y} \hat{x}_{k}\right)
\end{aligned}
$$

with

$$
\hat{x}_{k+1 / k}=A \hat{x}_{k}+B u_{k}+g\left(\hat{x}_{k}\right)
$$

\section{B. Extended UIO design for unknown input estimation}

In certain cases, it can be interesting to estimate the unknown inputs. Now, we propose the following extended UIO to estimate both system states and unknown inputs.

Consider the discrete-time Lipschitz system (26).

\section{Theorem 2:}

An extended UIO represented by the following equations

$$
\begin{aligned}
\hat{x}_{k+1}= & {\left[I-E\left(C_{y} E\right)^{+} C_{y}\right] \hat{x}_{k+1 / k}+E\left(C_{y} E\right)^{+} \bar{y}_{k+1} } \\
& +K\left(\bar{y}_{k}-C_{y} \hat{x}_{k}\right)
\end{aligned}
$$

with

$$
\hat{x}_{k+1 / k}=A \hat{x}_{k}+B u_{k}+g\left(\hat{x}_{k}\right)
$$

and

$$
\begin{gathered}
\hat{d}_{k+1}=\left(C_{y} E\right)^{+}\left[\bar{y}_{k+1}-C_{y} A \hat{x}_{k}-C_{y} B u_{k}-C_{y} g\left(\hat{x}_{k}\right)\right] \\
+K_{o}\left(C \hat{x}_{k}-y_{k}\right)
\end{gathered}
$$

where $K_{o} \in \Re^{q \times m}$ can be designed to reduce the estimation error bound.

Proof:

Form (26), we can write

$$
\bar{y}_{k+1}=C_{y} A x_{k}+C_{y} B u_{k}+C_{y} g\left(x_{k}\right)+C_{y} E d_{k}
$$

we then obtain

$$
d_{k}=\left(C_{y} E\right)^{+} \bar{y}_{k+1}-\left(C_{y} E\right)^{+} C_{y}\left[A x_{k}+B u_{k}+g\left(x_{k}\right)\right]
$$

Subsequently, we propose an estimator of the unknown inputs of the following form

$$
\hat{d}_{k+1}=\left(C_{y} E\right)^{+}\left(\bar{y}_{k+1}-C_{y} A \hat{x}_{k}-C_{y} B u_{k}-C_{y} g\left(\hat{x}_{k}\right)\right)
$$

Therefore

$$
\varphi_{k}=\hat{d}_{k+1}-d_{k}=\left(C_{y} E\right)^{+} C_{y}\left(A e_{k}+r_{k}\right)
$$

with

$$
e_{k}=x_{k}-\hat{x}_{k} \text { and } r_{k}=g\left(x_{k}\right)-g\left(\hat{x}_{k}\right)
$$

Using (2), we can conclude, if

$$
e_{k} \rightarrow 0
$$




$$
\hat{d}_{k+1} \rightarrow d_{k}
$$

Now, we propose to add to the equation (37) a correction term which depends on the output estimation error, where the new structure estimator can be written in the following form

$$
\begin{gathered}
\hat{d}_{k+1}=\left(C_{y} E\right)^{+}\left[\bar{y}_{k+1}-C_{y} A \hat{x}_{k}-C_{y} B u_{k}-C_{y} g\left(\hat{x}_{k}\right)\right] \\
+K_{o}\left(C_{y} \hat{x}_{k}-\bar{y}_{k}\right)
\end{gathered}
$$

with $K_{o} \in \Re^{q \times m}$ to reduce the estimation error.

The estimation error of unknown inputs can be expressed as

$\varphi_{k}=\hat{d}_{k+1}-d_{k}=\left(\left(C_{y} E\right)^{+} C_{y} A-K_{o} C_{y}\right) e_{k}+\left(C_{y} E\right)^{+} C_{y} r_{k}$

thus

$$
\left|\varphi_{k}\right| \geq\left\|\left(C_{y} E\right)^{+} C_{y} A-K_{o} C_{y}\right\| \cdot\left|e_{k}\right| \cdot\left\|\left(C_{y} E\right)^{+} C_{y}\right\| \cdot\left\|r_{k}\right\|
$$

such as

$$
\left\|r_{k}\right\|=\left\|g\left(x_{k}\right)-g\left(\widehat{x}_{k}\right)\right\| \leq \gamma\left\|x_{k}-\widehat{x}_{k}\right\| \rightarrow 0
$$

By minimizing $\varphi_{k}$, we obtain

$$
K_{o}=\left(C_{y} E\right)^{+}\left(C_{y} A C_{y}^{T}\right)\left(C_{y} C_{y}^{T}\right)^{-1}
$$

In conclusion, the unknown input estimation is given by

$$
\begin{aligned}
\hat{d}_{k+1}= & \left(C_{y} E\right)^{+}\left[\bar{y}_{k+1}-C_{y} A \hat{x}_{k}-C_{y} B u_{k}-C_{y} g\left(\hat{x}_{k}\right)\right] \\
& +K_{o}\left(C_{y} \hat{x}_{k}-\bar{y}_{k}\right)
\end{aligned}
$$

\section{ILLUSTRATIVE EXAMPLE}

The nonlinear model of a single link flexible joint robot arm is described by following set of equations [17]

$$
\left\{\begin{array}{l}
\dot{\theta}_{m}=\omega_{m} \\
\dot{\omega}_{m}=\frac{k}{J_{m}}\left(\theta_{l}-\theta_{m}\right)-\frac{B_{o}}{J_{m}} \omega_{m}+\frac{K_{\tau}}{J_{m}} u \\
\dot{\theta}_{l}=\omega_{l} \\
\dot{\omega}_{l}=-\frac{k}{J_{l}}\left(\theta_{l}-\theta_{m}\right)-\frac{m g h}{J_{l}} \sin \left(\theta_{l}\right)
\end{array}\right.
$$

where $\theta_{m}$ and $\omega_{m}$ are the position and angular velocity of the DC motor, respectively; $\theta_{l}$ and $\omega_{l}$ represent position and angular velocity of the link, the moment of inertia of the DC motor is denoted by $J_{m}$ while that of the link is denoted by $J_{l}$, the length of the link is given by $h$ while $m$ represents its mass, $B_{o}$ is the viscous friction, parameter $k$ symbolizes the torsional spring constant while $K_{\tau}$ is the amplifier gain.

The discrete-time system (25) was obtained by using the Euler discretisation of a step size $T_{e}=0.01$. The input signal was given by $u_{k}=\sin \left(2 \pi T_{e} k\right)$, the unknown input signal was given by $d_{k}=0.3 \sin \left(2 \pi T_{e} k+\frac{\pi}{3}\right)$, while the initial condition for the observer and the system were $\hat{x}_{0}=$ $\left[\begin{array}{cccc}1 & 0 & 0.5 & 0\end{array}\right]^{T}$ and $x_{0}=\left[\begin{array}{llll}0 & 0 & 0 & 0\end{array}\right]^{T}$, respectively. The flexible joint robot arm system can be described with system matrices

$$
A=\left[\begin{array}{cccc}
1 & 1 & 0 & 0 \\
-48.6 & -1.25 & 48.6 & 0 \\
0 & 0 & 0 & 10 \\
1.95 & 0 & -1.95 & 1
\end{array}\right], B=\left[\begin{array}{c}
0 \\
21.6 \\
0 \\
0
\end{array}\right]
$$

$$
\begin{gathered}
g\left(x_{k}\right)=\left[\begin{array}{c}
0 \\
0 \\
0 \\
-0.33 \sin \left(x_{3}\right)
\end{array}\right], E=\left[\begin{array}{c}
10 \\
20 \\
10 \\
0
\end{array}\right] \\
C=\left[\begin{array}{llll}
1 & 0 & 0 & 0 \\
0 & 1 & 0 & 0
\end{array}\right], F=\left[\begin{array}{c}
1 \\
-5
\end{array}\right]
\end{gathered}
$$

The Lipschitz constant of the nonlinear function is equal $\gamma=$ 0.0033. Solving the LMIs (6)-(7) condition of theorem 1 we obtain an observer gain

$$
\begin{gathered}
K=\left[\begin{array}{cc}
1.72 & -0.75 \\
-0.86 & 0.37 \\
-3.49 & 1.34 \\
-9.11 & 4.56
\end{array}\right] \\
P=\left[\begin{array}{cccc}
14.3393 & -0.3646 & 5.9215 & -0.8769 \\
-0.3646 & 13.7924 & -2.9607 & 0.4384 \\
5.9215 & -2.9607 & 7.9389 & -2.0037 \\
-0.8769 & 0.4384 & -2.0037 & 0.5929
\end{array}\right]
\end{gathered}
$$

The following maximum value of $\gamma$ was obtained for the design procedure: $\gamma=0.0155$. This means that the Lipschitz constant $\gamma$ is acceptable.

If the unknown inputs will simultaneously affect the state and the measurement equation we can find these simulation results:

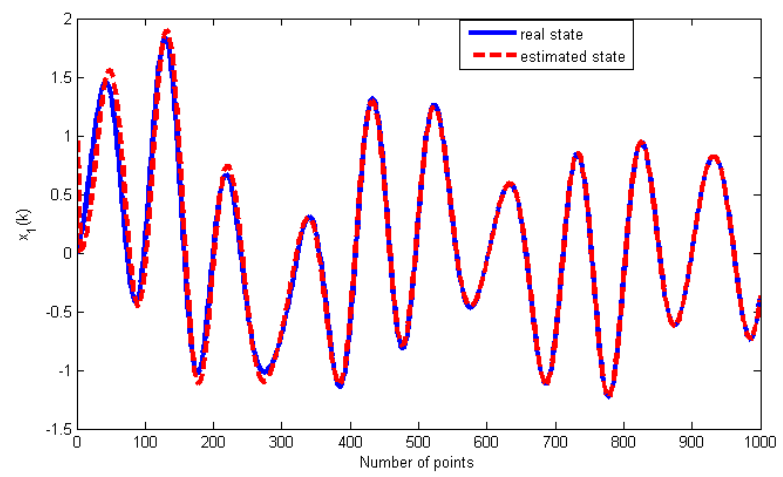

Fig. 1. Angular position of the motor $x_{1}$ and its estimates.

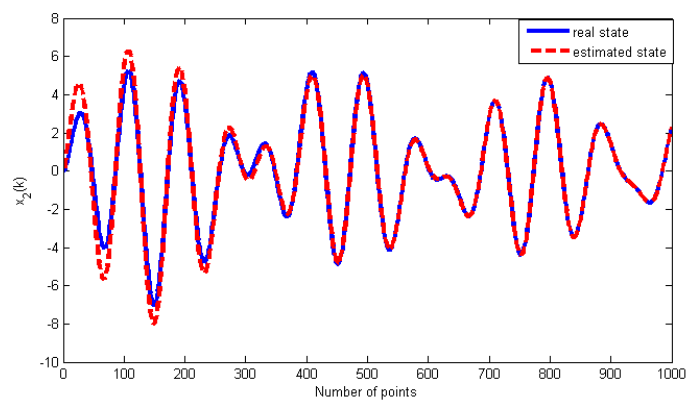

Fig. 2. Angular velocity of the motor $x_{2}$ and its estimates. 


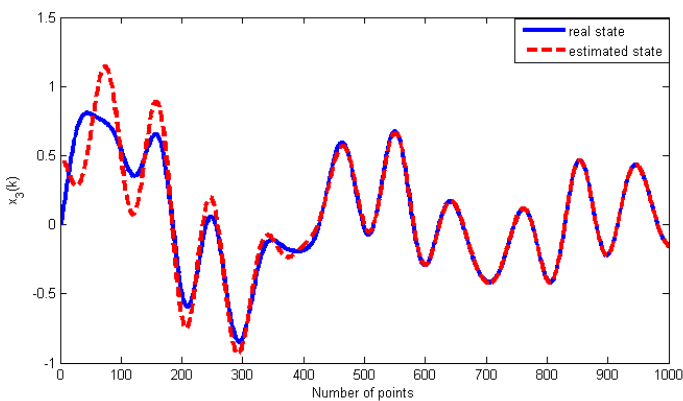

Fig. 3. Angular position of the link $x_{3}$ and its estimates.

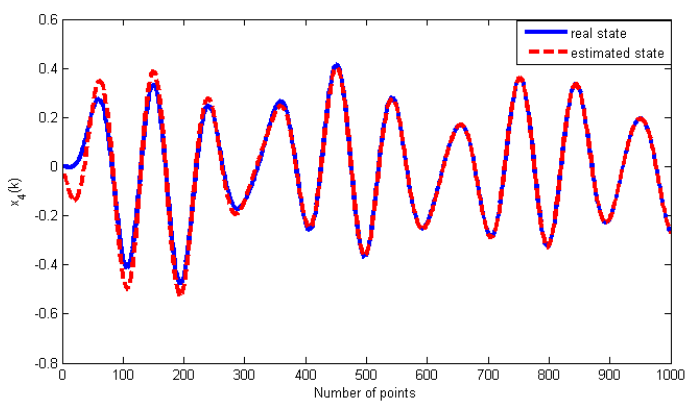

Fig. 4. angular velocity of the link $x_{4}$ and its estimates.

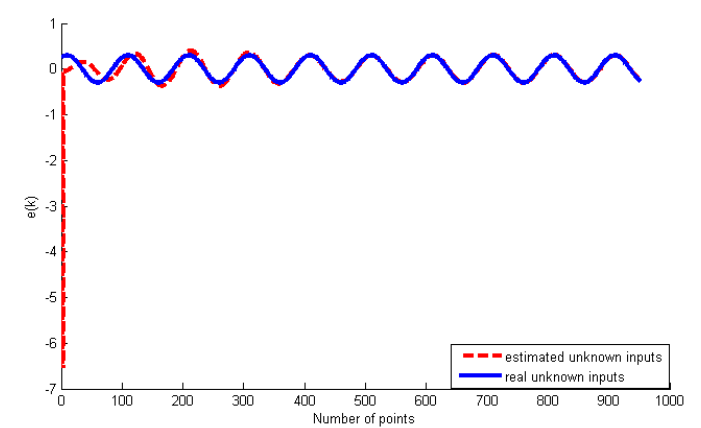

Fig. 5. The unknown input real and its estimates.

\section{CONCLUSION}

In this paper, we developed a design method of the unknown inputs observers design for a class of Lipschitz nonlinear discrete-time systems was addressed. This method is based on one procedure applied to extended unknown input observers that use a well determined design algorithm. In addition, this procedure enables to increase the variation of the Lyapunov function candidate using the technique of singular values.

\section{REFERENCES}

[1] M. Darouach, Complements to full order observers design for linear systems with unknown inputs, Applied Mathematics Letters, vol. 22, no. 7, pp. 1107-1111, 2009.
[2] M. Darouach, On the level approach to the design of unknown input observers, IEEE Transaction on Automatic Control, vol. AC-39, no. 3, March, pp. 698-699, 1994.

[3] S. K. Chang and P. L. Hsu, Fault detection observer design for linear systems with unknown inputs, In Proc.ECC conference, Groningen, the Netherlands, 1993.

[4] Y. Guan and M. Saif, A novel approach to design of unknown-input observer, IEEE Transaction on Automatic Control, vol. 36, pp. 631-635, 1991.

[5] J. E. Kurek, The state vector reconstruction for linear systems with unknown inputs, IEEE Transaction on Automatic Control, vol. AC-28, pp. 1120-1122, 1983.

[6] F. Yand and R. W. Wilde, Observers for linear systems with unknown inputs, IEEE Transaction on Automatic Control, vol. 33, pp. 677-681, 1988.

[7] P. Kudva, N. Viswanadham and A. Ramakrishna, Observers for linear systems with unknown inputs, IEEE Transaction on Automatic Control, vol. AC- 25, pp. 113-115, 1980.

[8] M. E. Valcher, State observer for discrete-time linear systems with unknown inputs, IEEE Transaction on Automatic Control, vol. 44, 1999.

[9] F. Yang and R.W. Wilde, Observers for linear systems with unknown inputs, IEEE Transaction on Automatic Control, vol. 33, pp. 677-681, 1998.

[10] J. Wunnenberg, Observer-based fault detection in dynamic systems, Phd thesis Dusseldof, Germany, 1990.

[11] P. M. Frank, Fault diagnosis in dynamic systems using analytical and knowledge-based redundancy-A survey and some new results, Automatica, vol. 26, pp. 459-474, 1990.

[12] F. Thau, Observing the state of nonlinear dynamic Systems, International Journal Control, vol. 17, no. 3, pp. 471-479, 1973.

[13] M. S. Chen and C. C. Chen, Robust nonlinear observer for Lipschitz nonlinear systems subject to disturbances, IEEE Transaction on Automatic Control, vol. 52, no. 12, pp. 2365-2369, 2007.

[14] M. Pertew, H. marquez. and Q. Zhao, H1 observer design for Lipschitz nonlinear systems, IEEE Transaction on Automatic Control, vol. 51, no. 7, pp. 1211-1216, 2006.

[15] M. Abbaszadeh and H. Marquez, A robust observer design method for continuous-time Lipschitz nonlinear systems, Proceeding 45th IEEE Conference on Decision and Control, pp. 3795-3800, 2006.

[16] S. Raghavan and J. Hedrick, Observers design for a class of nonlinear systems, International Journal Control, vol. 59, pp. 515-528, 1998.

[17] R. Rajamani, Observers for Lipschitz nonlinear systems, IEEE Transaction on Automatic Control, vol. 43, no. 3, pp. 397-401, 1988.

[18] A. Zemouche, M. Boutaye and G. Bara, Observers for a class of Lipschitz systems with extension to $\mathrm{H} 1$ performance analysis., Systems and Control Letters, vol. 57, pp. 18-27, 2008.

[19] A. Zemouche and M. Boutayeb, Nonlinear observers for Lipschitz discrete-time systems, European Control Conference ECC07, Kos, Greece, July, 2007.

[20] K. Busawon, M. Saif and M. Farza, A discrete-time observer for a class of nonlinear systems, In Proc 36 the IEEE Conference on Decision and Control CDC, pp. 4796-4801, 1997.

[21] Z. Wang and Unbehauen, A class of non-linear observes for discrete-time systems with parametric uncertainty, International Journal of Systems Science, vol. 31, no. 1, pp. 177-221, 2000.

[22] S. Xu, Robust H filtering for a class of discrete-time uncertain nonlinear systems with state delay, IEEE Transactions on Circuits and SystemsI: Fundamental Theory and Applications, vol. 49, no. 12, pp. 1853-1859, 2002.

[23] Q. P. Ha and H. Trinhb, State and input simultaneous estimation for a class of nonlinear systems, Automatica, vol. 40, pp. 1779-1785, 2004.

[24] M. Witczak, Modeling and Estimation Strategies for Fault Diagnosis of Non-Linear Systems, Springer-Verlag Berlin Heidelberg, 2007. 